\title{
Differentiation-related zinc finger protein 750 suppresses cell growth in esophageal squamous cell carcinoma
}

\author{
SHEYNE STA ANA CHOI, JOSEPHINE MUN-YEE KO, VALEN ZHUOYOU YU, \\ LVWEN NING and MARIA LI LUNG
}

Department of Clinical Oncology, The University of Hong Kong, Hong Kong, SAR, P.R. China

Received November 8, 2020; Accepted March 12, 2021

DOI: 10.3892/ol.2021.12774

\begin{abstract}
Esophageal squamous cell carcinoma (ESCC) is a deadly squamous cell carcinoma (SCC) of the esophagus. Development of SCCs is associated with the deregulation of the squamous cell lineage program and/or keratinocyte terminal differentiation by genomic and genetic aberrations; thus, these processes must be tightly controlled to maintain normal squamous cell development. Zinc finger protein 750 (ZNF750) is a gene involved in keratinocyte terminal differentiation and is frequently mutated and putatively silenced in ESCC, which implicates its function as a potential differentiation-related suppressor of ESCC. The present study aimed to elucidate the relationship between ZNF750 function to induce keratinocyte differentiation and tumor suppression in ESCC. The results demonstrated that chemical manipulation of esophageal keratinocyte differentiation in mouse normal esophageal epithelial organoids (mNEEO) implicated the involvement of the mouse homologue of ZNF750, Zfp750, in keratinocyte differentiation in premalignant cells. Bioinformatics analyses of data from high ZNF750-expressing ESCC tumors obtained from public databases and ZNF750-overexpressing ESCC cells compared with low ZNF750-expressing ESCC tumors and GFP-expressing ESCC cells, respectively, revealed enrichment of keratinocyte differentiation-related gene sets in these samples. Finally, the induction through to terminal differentiation of the keratinocyte by all-trans retinoic acid on parental ESCC cell lines led to the upregulation of the terminal differentiation marker Involucrin and a decrease in cell viability similar to that observed in ZNF750-overexpressing ESCC cells. The results of the present study demonstrated a functional link between the ability of ZNF750 to induce cell differentiation through to terminal differentiation and its
\end{abstract}

Correspondence to: Professor Maria Li Lung, Department of Clinical Oncology, The University of Hong Kong, 21 Sassoon Road, Pokfulam, Hong Kong, SAR, P.R. China

E-mail: mlilung@hku.hk

Key words: esophageal squamous cell carcinoma, zinc finger protein 750 , keratinocyte differentiation, squamous cell carcinoma, tumor suppressor gene function as a growth suppressor in ESCC. This study provides improved understanding of the role of ZNF750, a frequently mutated differentiation-related gene in ESCC, and its effects in ESCC pathogenesis.

\section{Introduction}

Esophageal cancer is a deadly type of cancer that ranks 8th in terms of worldwide cancer mortality (1). The predominant histological subtype in most parts of the world, especially in East and Central Asia and parts of East Africa and South Africa, is esophageal squamous cell carcinoma (ESCC) (2). The development of squamous cell carcinomas (SCCs) is associated with various genomic and genetic aberrations that lead to the deregulation of the squamous cell lineage and terminal differentiation program; thus, these processes must be tightly controlled to maintain normal squamous epithelium development (3).

Zinc finger protein 750 (ZNF750) is a member of the zinc finger family proteins, which are involved in numerous biological processes, including keratinocyte and epithelial cell differentiation, as well as adipogenesis (4). These proteins have also been implicated in different diseases including diabetes, psoriasis and cancer $(4,5)$. ZNF750 has been reported to be involved in the maintenance of epithelial homeostasis and the epidermal terminal differentiation process in primary and organotypic human keratinocyte culture through its interaction with transcription factors and epigenetic modifiers $(6,7)$. In addition, knockout of ZNF750 in squamous cells silenced the expression levels of differentiation-related genes Krüppel-like factor 4, S100 calcium-binding protein A8 and loricrin (8). Frequent somatic mutations within the exonic regions have been reported in cervical, head and neck, lung and esophageal SCCs (7-9), implicating the silencing of ZNF750 in these malignancies.

The whole-exome sequencing analysis in our previous study demonstrated the clinical relevance of ZNF750 in ESCC (10). ZNF750 levels are downregulated in ESCC tumors compared with those in distant non-tumor tissues, and mutations in this gene are significantly more frequent in primary ESCC tumors with lymph node (LN) metastasis compared with those without LN metastasis (10). The levels of differentiation-related genes, including small proline-rich protein 3 (SPRR3) and transglutaminase 3 (TGM3), have been reported to be downregulated 
in primary tumor samples compared with those in adjacent non-tumor tissues $(11,12)$. In addition, SPRR3 exhibits tumor-suppressive functions in ESCC cells (13), indicating a link between silenced differentiation-related genes and tumor-suppressive functions in ESCC. Therefore, we hypothesized that ZNF750 as a frequently mutated and downregulated differentiation-related gene may function similarly to other downregulated differentiation-related genes, such as SPRR3, and act as a tumor suppressor in ESCC.

The function of ZNF750 in the suppression of SCCs, including ESCC, has been demonstrated in an in vitro cell viability and an in vivo tumorigenesis models $(9,14)$, whereas its functions in keratinocyte differentiation induction have been reported in keratinocyte cell line and organoid models (5-7); however, the functional link between keratinocyte differentiation induction and tumor suppression in ESCC has not been fully explored. The aim of the present study was to elucidate this relationship.

\section{Materials and methods}

Cell culture. The KYSE-series cell lines (ACC 351, ACC 375, ACC 379, ACC 363, ACC 374 ACC 380, ACC 387; DSMZ) were cultured in RPMI-1640 medium (cat. no. 23400) supplemented with $10 \%$ fetal bovine serum (FBS; cat. no. 10270) and $1 \%$ penicillin-streptomycin (cat. no. 15070). NE-1 cells were cultured in Defined Keratinocyte SFM medium with Defined Keratinocyte SFM Growth Supplement (cat. no. 10744019). 293T, HKESC-2 and SLMT cells were cultured in DMEM (cat. no. 12100) supplemented with 5\% FBS and 5\% Newborn Calf Serum (NCS; cat. no. 16010), and 1\% penicillin-streptomycin. 293T cells served as host cells for lentiviral production. Cells were seeded and incubated until full confluence and passaged every 2-3 days using Trypsin/EDTA (cat. no. 25200) for continuous culture at $37^{\circ} \mathrm{C}$ with $5 \% \mathrm{CO}_{2}$. All reagents used for cell culture were purchased from Invitrogen; Thermo Fisher Scientific, Inc. The cell lines were genotyped, authenticated and tested for mycoplasma prior to the experiments.

Mouse normal esophageal epithelial organoid (mNEEO) generation and culture. Mouse esophageal tissue isolation, organoid generation and culture were performed as previously described with modifications (15). In brief, 8-10-week old BALB/c/nu/nu athymic mice $(n=4)$ were sacrificed through anesthetic overdose by intraperitoneal injection of ketamine/xylazine $(300 \mathrm{mg} / \mathrm{kg}$ ketamine and $30 \mathrm{mg} / \mathrm{kg}$ xylazine) followed by carbon dioxide asphyxiation by placing mice in a carbon dioxide-filled container. The esophagus was surgically removed, and the mucosa was pulled away from the submucosa layer using forceps. The mucosa layer was cut into small pieces with a scalpel, dissociated in Trypsin-EDTA at $37^{\circ} \mathrm{C}$ for $30 \mathrm{~min}$ and subsequently neutralized with RPMI-1640 supplemented with $10 \%$ FBS. To generate organoids, the dissociated cells were suspended in Matrigel ${ }^{\circledR}$ (cat. no. 356234; Corning, Inc.) and placed in 24-well low-bind culture plates (cat. no. 174930; Thermo Fisher Scientific, Inc.) at $37^{\circ} \mathrm{C}$ to solidify. The mNEEO was cultured in growth medium comprising advanced DMEM/F12 (cat. no. 12634), $1 \mathrm{X}$ N2 (cat. no. 17502) and 1X B27 Supplements (cat. no. 17504), 1X Glutamax (cat. no. 35050), 1X HEPES (cat. no. 15630),
$1 \mathrm{X}$ penicillin/streptomycin, $1 \mathrm{mM} \mathrm{N}$-acetyl-L-cysteine (cat. no. A9165, Sigma-Aldrich; Merck KGaA), $100 \mu \mathrm{m}$ gastrin (cat. no. G9020, Sigma-Aldrich; Merck KGaA), 10 mM nicotinamide (cat. no. N0636, Sigma-Aldrich; Merck KGaA), $10 \mu \mathrm{m}$ SB202190 (cat. no. S7067, Sigma-Aldrich; Merck KGaA), $50 \mathrm{ng} / \mathrm{ml}$ epidermal growth factor (cat. no. PHG0314), $100 \mathrm{ng} / \mathrm{ml}$ Noggin (cat. no. 120-10C; PeproTech, Inc.), 100 ng/ml Wnt3A (cat. no. 315-20; PeproTech, Inc.), 100 ng/ml R-Spondin 2 (cat. no. 120-43; PeproTech, Inc.) and 500 nM A8301 (cat. no. 2939; Tocris Bioscience) as previously described (15) and passaged every 7 days for continuous culture. All reagents were supplied by Thermo Fisher Scientific, Inc. unless otherwise specified. Ethics approval for animal studies was obtained from the Animal Ethics Committee of The University of Hong Kong (approval no. CULATR 3377-14).

Chemically induced manipulation of mNEEO differentiation. For all-trans retinoic acid (ATRA; cat. no. R2625; Sigma-Aldrich; Merck KGaA) and Compound E (cat. no. sc-221433; Santa Cruz Biotechnology, Inc.) treatment of mNEEO, 2,000 dissociated esophageal epithelial cells were embedded in 4 wells/group in a low-bind 96-well culture plate (cat. no. 15237905; Thermo Fisher Scientific, Inc.). The esophageal epithelial cells were cultured in growth medium for $24 \mathrm{~h}$ prior to ATRA and Compound $\mathrm{E}$ treatment. Growth medium was replaced with growth medium containing $100 \mathrm{nM}$ ATRA, $10 \mu \mathrm{M}$ Compound E or DMSO (cat. no. D418; Sigma-Aldrich; Merck KGaA) vehicle after $24 \mathrm{~h}$, and the cells were cultured for 9 days to generate organoids.

Molecular cloning of ZNF750. ZNF750 (NM_024702.3) was amplified using Q5 High-Fidelity DNA Polymerase (cat. no. M0491; New England Biolabs, Inc.) from KYSE30 cell line cDNA by PCR. The PCR procedure was performed using a Veriti $^{\text {TM }}$ 96-Well Thermal Cycler (cat. no. 4375786; Thermo Fisher Scientific, Inc.) under the following cycling conditions: Initial denaturation at $98^{\circ} \mathrm{C}$ for $30 \mathrm{sec}$, followed by 30 cycles of annealing at $56^{\circ} \mathrm{C}$ for $30 \mathrm{sec}$ and extension at $72^{\circ} \mathrm{C}$ for $2 \mathrm{~min}$. The oligonucleotide pairs used for amplification were $Z N F 750$ forward, 5'-GGAATTCGCCACCATGAGTCTCCTCAAAGA GCGG-3' and reverse, 5'-GCTCTAGATTACTTGTCGTCA TCGTCTTTGTAGTCGGACACCCGGGCCCTC-3'. The amplifiedDNA wassubsequentlycloned into apLVX-EF1 $\alpha$-puro vector. The ZNF750 sequence (pLVX-ZNF750) was validated by Sanger sequencing (16) at the Centre for PanorOmic Sciences (CPOS) at the University of Hong Kong (Hong Kong, SAR, China). pLVX-puro (cat. no. 632164; Clontech Laboratories, Inc.) was modified by removing the CMV promoter and replacing it with an EF1 $\alpha$ promoter to construct a pLVX-EF1 $\alpha$-puro vector (17). The pLVX-EF1 $\alpha$-puro vector was used to insert a GFP gene to create the pLVX-GFP control plasmid (17).

Lentivirus production and infection. 293T cells were seeded in preparation for viral production in T25 culture flasks. When 293 T cells reached $60 \%$ confluence, the transfection mixture was prepared using $8 \mu \mathrm{l}$ X-tremeGENETM $\mathrm{HP}$ DNA Transfection Reagent (cat. no. 6366546001; Roche Molecular Systems, Inc.), $2 \mu \mathrm{g}$ plasmid DNA (pLVX-GFP or pLVX-ZNF750), $1.5 \mu \mathrm{g}$ viral packaging vector psPAX2 (cat. no. 12260; Addgene, Inc.) and $0.5 \mu \mathrm{g}$ viral envelope vector 
pMD2.G (cat. no. 12259; Addgene, Inc.) in $400 \mu 1$ serum-free DMEM and incubated for $20 \mathrm{~min}$ at room temperature. The transfection mixture was added dropwise to the culture flask of HEK293T cells and incubated for $72 \mathrm{~h}$ at $37^{\circ} \mathrm{C}$. Subsequently, the viral supernatant was passed through a $0.45-\mu \mathrm{m}$ filter and aliquoted. The virus was stored at $-80^{\circ} \mathrm{C}$ until further experiments. For lentiviral infection, KYSE30 and KYSE180 cells were seeded to achieve a confluency of $\sim 30 \%$ at $24 \mathrm{~h}$ post-seeding. A $1 \mathrm{ml}$ aliquot of pre-prepared virus expressing GFP or ZNF750 with $0.006 \mu \mathrm{l} 5 \mu \mathrm{g} / \mathrm{ml}$ polybrene (cat. no. H9268; Sigma-Aldrich; Merck KGaA) was added to cells in tissue culture flasks to facilitate lentiviral infection. The medium was changed at $24 \mathrm{~h}$ post-infection to allow the cells to recover. The cells were passaged at day 2 post-infection for expansion and prepared for assays after the second passage.

RNA isolation, reverse transcription $(R T)$ and quantitative (q)PCR. RNA was extracted from cells and organoids using the TRI Reagent ${ }^{\mathrm{TM}}$ solution (cat. no. AM9738; Invitrogen; Thermo Fisher Scientific, Inc.), according to the manufacturer's instructions and reverse-transcribed to complementary DNA using PrimeScript RT Master Mix (Perfect Real Time) (cat. no. RR036; Takara Bio, Inc.) according to the manufacturer's instructions. FastStart Universal SYBR ${ }^{\circledR}$-Green Master (Rox) (cat. no. 4913914001; Roche Life Science) was used to determine the mRNA expression levels of target genes. Each reaction comprised 1X FastStart Universal SYBR ${ }^{\circledR}$ Green Master mix, $30 \mathrm{ng} / \mu 1 \mathrm{cDNA}$, and $0.4 \mathrm{mM}$ each of forward and reverse primer. The reactions were performed in the LightCycler $^{\circledR} 480$ Instrument II (cat. no. 05015243001; Roche Life Science) using the default SYBR ${ }^{\circledR}$-Green program. mRNA expression levels were quantified using the $2^{-\Delta \Delta \mathrm{Cq}}$ method (18). Human GAPDH forward, 5'-AAGGTGAAGGTCGGAGTC-3' and reverse, 5'-GAAGATGGTGATGGGATTTC-3', was used as the internal control. The primer sequences were as follows: Human: ZNF750 forward, 5'-AAAGCACAGAATGCCTAC CTG-3' and reverse, 5'-GGGTCTCCGTTCACAACATT-3'; keratin 4 (KRT4) forward, 5'-GATGCTCTTCTGGGGGAT T-3' and reverse, 5'-GCTCTGGTTGATGGTGACCT-3'; SPRR1A forward, 5'-CCGTATACCAGCTTTCTGTCTC-3' and reverse, 5'-GCTGGCAAGGTTGTTTCAC-3'; SPRR2E forward, 5'-TGGTACTTGAGCACTGATCTGC-3' and reverse, 5'-TGCACTGCTGCTGTTGATAA-3'; TGM3 forward, 5'-TGG AAGGACTCTGCCACAAT-3' and reverse, 5'-TGAGCG TACGAGATCTTTATGG-3'; and involucrin (IVL) forward, 5'-GCAGGAGGGACAGCTGAA-3' and reverse, 5'-ATCTGC TCCTCTGGGACCTC-3'; mouse: Zfp750 forward, 5'-GAG CCAGCGTGAGAACAGA-3' and reverse, 5'-CAGGAGAGT TCCTTCCGTCA-3'; $I v l$ forward, 5'-CCCTAGTTGCTGCTC AGCTC-3' and reverse, 5'-TGGAAGCTTCTGTAGACCAAA A-3'; Krt4 forward, 5'-AACAAATTCGCGTCCTTCAT-3' and reverse, 5'-CTGCTGGAGGAGGTTCCAT-3'; and Krt14 forward, 5'-ATCGAGGACCTGAAGAGCAA-3' and reverse, 5'-TCGATCTGCAGGAGGACATT-3'.

Protein extraction and western blotting. Protein lysates were obtained by lysing KYSE30 and KYSE180 cells for $10 \mathrm{~min}$ at $4^{\circ} \mathrm{C}$ in RIPA buffer (cat. no. 89900; Thermo Fisher Scientific, Inc.) supplemented with $1 \mathrm{X}$ Halt ${ }^{\mathrm{TM}}$ Protease and Phosphatase Inhibitor Cocktail (cat. no. 78442; Thermo Fisher Scientific,
Inc.), and 1 unit of Pierce Universal Nuclease (cat. no. 88700; Thermo Fisher Scientific, Inc.). Protein lysates were collected by centrifugation at $12,000 \mathrm{x}$ g at $4^{\circ} \mathrm{C}$ for $10 \mathrm{~min}$. The proteins were denatured at $95^{\circ} \mathrm{C}$ for $10 \mathrm{~min}$ prior to electrophoresis. A total of $50 \mu \mathrm{g}$ protein per sample were electrophoresed using 12\% SDS-PAGE at $80 \mathrm{~V}$ for $30 \mathrm{~min}$ and $120 \mathrm{~V}$ for $1.5 \mathrm{~h}$ using the Mini Gel Tank (cat. no. A25977; Thermo Fisher Scientific, Inc.). Following electrophoresis, the proteins within the gel were transferred to methanol-activated $0.45-\mu \mathrm{m} \mathrm{PVDF}$ membranes (cat. no. IPVH00010; MilliporeSigma) using a Mini Blot system (cat. no. B1000; Thermo Fisher Scientific, Inc.) at $30 \mathrm{~V}$ for $1 \mathrm{~h}$. Subsequently, the membranes were blocked with $3 \%$ bovine serum albumin (v/v) in Tris-buffered saline with $0.1 \%$ Tween (TBST) for $45 \mathrm{~min}$ at room temperature. The membranes were hybridized with the primary antibodies at $4^{\circ} \mathrm{C}$ overnight with constant shaking. The membranes were briefly washed with TBST and probed with a horseradish peroxidase (HRP)-conjugated secondary antibody diluted 1:5,000 for $1 \mathrm{~h}$ at room temperature with gentle agitation, followed by extensive washing with TBST prior to signal detection. Signals were detected by WesternBright ECL HRP substrate (cat. no. K-12045; Advansta, Inc.) and Super RX X-ray films (Fujifilm Corporation). Band intensities were measured using ImageJ software version 1.53a (National Institutes of Health) (19) and quantified by normalization to the GFP control. The primary antibodies used were polyclonal rabbit anti-human ZNF750 (1:1,000; cat. no. 21752-1-AP; Proteintech Group, Inc.), polyclonal rabbit anti-human SPRR3 (1:1,000; cat. no. A12041; ABclonal, Inc.), monoclonal mouse anti-human IVL (1:1,000; cat. no. MA5-11803; Thermo Fisher Scientific, Inc.), monoclonal mouse anti-human p84 (1:1,000; cat. no. GTX70220; GeneTex, Inc.), monoclonal rabbit anti-human GAPDH (1:5,000; cat. no. 5174; Cell Signaling Technology, Inc.) and monoclonal rabbit anti-GFP $(1: 1,000$; cat. no. 2956; Cell Signaling Technology, Inc.). The secondary antibodies used were polyclonal anti-mouse $\operatorname{IgG}$ (1:5,000; cat. no. GTX213111-01) and polyclonal anti-rabbit IgG (1:5,000; cat. no. GTX213110-01) (both from GeneTex, Inc.).

RNA sequencing (RNA-seq) and transcriptomic analysis of ZNF750-upregulation in ESCC cells. Total RNAs of pLVX-GFP- or pLVX-ZNF750-transduced KYSE180 cells were extracted with the AllPrep DNA/RNA Micro kit (cat. no. 80284; Qiagen, Inc.) according to the manufacturer's instructions for RNA sequencing. Library preparation and Illumina sequencing (paired-end sequencing of $151 \mathrm{bp}$ ) were performed at CPOS. Bioanalyzer RNA Nano 6000 (Agilent Technologies) was used to determine the quality of the RNA. Ribosomal RNA (rRNA) depletion was performed using NEBNext ${ }^{\circledR}$ rRNA Depletion Kit (Human/Mouse/Rat) (cat. no. E6310; New England BioLabs, Inc.). cDNA libraries were prepared using the NEBNext ${ }^{\circledR}$ Ultra II Directional RNA Library Prep Kit (cat. no. E7760; New England BioLabs,Inc.). A total of $500 \mathrm{ng}$ RNA was used as starting material for ribosomal RNA depletion. Cytoplasmic and mitochondrial rRNA were firstly hybridized to ssDNA capture probes and then digested by Ribonuclease H. Subsequently, the residual ssDNA probes were digested by DNase I. The rRNA-depleted RNA was purified by Agencourt ${ }^{\circledR}$ RNAClean ${ }^{\mathrm{TM}}$ XP beads (cat. no. A63987; Beckman Coulter, Inc.). The purified rRNA-depleted RNA was 
fragmented to $200-500 \mathrm{bp}$ by incubating at $94^{\circ} \mathrm{C}$ for $10 \mathrm{~min}$ in the presence of magnesium ions (NEBNext ${ }^{\circledR}$ Magnesium RNA Fragmentation Module; cat no. E6150; New England BioLabs, Inc.). The fragmented rRNA-depleted RNA was then applied as a template to synthesize the first-strand cDNA using random hexamer-primer and a reverse transcriptase. In the second strand cDNA synthesis, the mRNA template was removed, and a replacement strand was generated to form the blunt-end double-stranded (ds) cDNA. The ds cDNA underwent 3'adenylation and indexed adaptor ligation, followed by treatment with User Enzyme. The adaptor-ligated libraries were enriched and indexed using NEBNext ${ }^{\circledR}$ Multiplex Oligos for Illumina ${ }^{\circledR}$ (96 Unique Dual Index Primer Pairs) (cat. no. E6440; New England BioLabs, Inc.) by 8 cycles of PCR according to the manufacturer's instructions. The concentration was determined using qPCR following library construction, and the libraries were denatured and diluted to $1.2 \mathrm{nM}$. Illumina NovaSeq 6000 Reagent Kit (cat. no. 20028312; Illumina, Inc.) was used for paired-end 151bp sequencing. Raw RNA-Seq data were cleaned and aligned to the hg19 reference genome using Tophat (version 2.0.14; bowtie version 2.2.4) (20) with library-type fr-firststrand parameter. Gene expression profile and differentially expressed genes were calculated by Cufflinks (version 2.2.1) with the Ensemble gene annotation file and Cuffdiff (21), respectively. Differential gene expression was calculated by $\log _{2}$ fold-change (FC) expression. Genes with $\log _{2}(\mathrm{FC}) \geq 2$ were used for further analysis.

Determination of enriched pathways in ZNF750-upregulated ESCC cells. Gene Set Enrichment Analysis (GSEA) (22) and ToppFun (https://toppgene.cchmc.org/enrichment. jsp) (23) were used for the analysis of enriched pathways in ZNF750-overexrpressing cells. Using GSEA version 4.1.0, transcriptomic data were analyzed to identify the enriched gene sets in the ZNF750-overexpressing group compared with the GFP-expressing group to identify the genes driving the enrichment. In ToppFun, differentially upregulated genes in the ZNF750-overexpressing group compared with the control group were used for analysis. The gene sets from the Molecular Signatures Database (MsigDB; http://www.broad. mit.edu/gsea/msigdb/index.jsp) were used for both analyses. The consensus gene sets between the two analyses with a false discovery rate (FDR) $\leq 0.25$ were considered to be enriched.

Extraction and analysis of the cancer genome atlas (TCGA) esophageal carcinoma (ESCA) RNA-seq data. TCGA data were downloaded from the National Cancer Institute Genomic Data Commons (https://gdc.cancer.gov/) (24). Normalized gene expression profiles of SCC samples with RNA-seq data from the TCGA-ESCA project (dbGaP study accession no. phs000178) (25) were extracted using $\mathrm{R}$ version 4.0.2 (26) and the TCGAbiolinks R/Bioconductor package (27) (http://bioconductor.org/packages/release/bioc/html/TCGAbiolinks. html). ESCC data were divided into high-ZNF750 expressing tumors (ZNF750-high; n=40) and low-ZNF750 expressing tumors ( $Z N F 750$-low; $\mathrm{n}=41$ ) using median normalized expression levels as the cut-off value. GSEA was used to determine the enriched gene sets in the ZNF750-high compared with the ZNF750-low group using the same cut-off values as aforementioned.
MTT cell viability assay. To assess cell viability, $2.5 \times 10^{3}$ KYSE30 and $5 \times 10^{3}$ KYSE180 cells per well were seeded in a 96-well plate with $100 \mu \mathrm{l}$ medium. For visualization, $30 \mu \mathrm{l} /$ well $5 \mathrm{mg} / \mathrm{ml}$ MTT dye (cat. no. M6494; Thermo Fisher Scientific, Inc.) was added to the cells and incubated at $37^{\circ} \mathrm{C}$ for $2 \mathrm{~h}$. Subsequently, the medium was aspirated, and $100 \mu \mathrm{l}$ DMSO was added to dissolve the formazan crystals. Absorbance was measured at $540 \mathrm{~nm}$ using an Ao Absorbance Microplate Reader (Azure Biosystems) daily for 3 days post-seeding.

ATRA treatment of parental ESCC cell lines. For the ATRA treatment, parental KYSE30 and KYSE180 cells were seeded as aforementioned and cultured for $24 \mathrm{~h}$. Subsequently, the culture medium in each well was replaced with medium containing 50 or $100 \mathrm{nM}$ ATRA or DMSO and cultured for 3 days. Concurrently, cells from the same batch were seeded in T25 culture flasks and treated with ATRA. RNA was extracted as aforementioned from the ATRA-treated cells at $48 \mathrm{~h}$ post-treatment and subjected to RT-qPCR analysis. Experiments were performed on KYSE30 and KYSE180 cell lines using previously reported ATRA concentrations as a reference to determine the optimal ATRA concentration for each cell line (28).

Colony formation assay. For the colony formation assay, $1 \times 10^{3}$ KYSE30 or KYSE180 cells were seeded into a single well of a 6-well culture plate to form colonies. The colonies were fixed with $37 \%$ formaldehyde for $30 \mathrm{~min}$ at room temperature and stained with 1:20 Giemsa solution in water overnight at room temperature. The solution was washed away with $1 \mathrm{X}$ PBS until the buffer was clear. Colonies were counted using the Gel Doc XR+ system (cat. no. 170-8195; Bio-Rad Laboratories, Inc.).

Subcutaneous tumor mouse model. ESCC tumorigenicity was evaluated by subcutaneous inoculation of ESCC cells into the flanks of 8-10-week-old BALB/c/nu/nu athymic nude mice. A total of $1 \times 10^{6} \mathrm{KYSE} 30$ cells were resuspended in $150 \mu \mathrm{l}$ of serum-free medium and injected in both flanks of each mouse ( $n=4$ mice per group). Tumor growth was monitored weekly for $\geq 4$ weeks and measured by calipers to calculate the tumor volume as follows: Volume=(length $\mathrm{x}$ width $\mathrm{x}$ height). At the experimental endpoint, mice were sacrificed by anesthetic overdose through intraperitoneal injection of ketamine/xylazine $(300 \mathrm{mg} / \mathrm{kg}$ ketamine and $30 \mathrm{mg} / \mathrm{kg}$ xylazine) followed by carbon dioxide asphyxiation by placing mice in a carbon dioxide-filled container. Cessation of cardiac function was used as the method for verification of death. All experimental procedures were approved by the Committee on the Use of Live Animals in Teaching and Research at The University of Hong Kong (approval no. CULATR 3377-14).

Statistical analysis. Data are presented as the mean \pm SEM. Each in vitro experiment was repeated at least three times in duplicate, unless otherwise stated. Unpaired Student's t-test was performed for all statistical analyses using GraphPad Prism version 8.4.3 (GraphPad Software, Inc.). $\mathrm{P}<0.05$ was considered to indicate a statistically significant difference. 

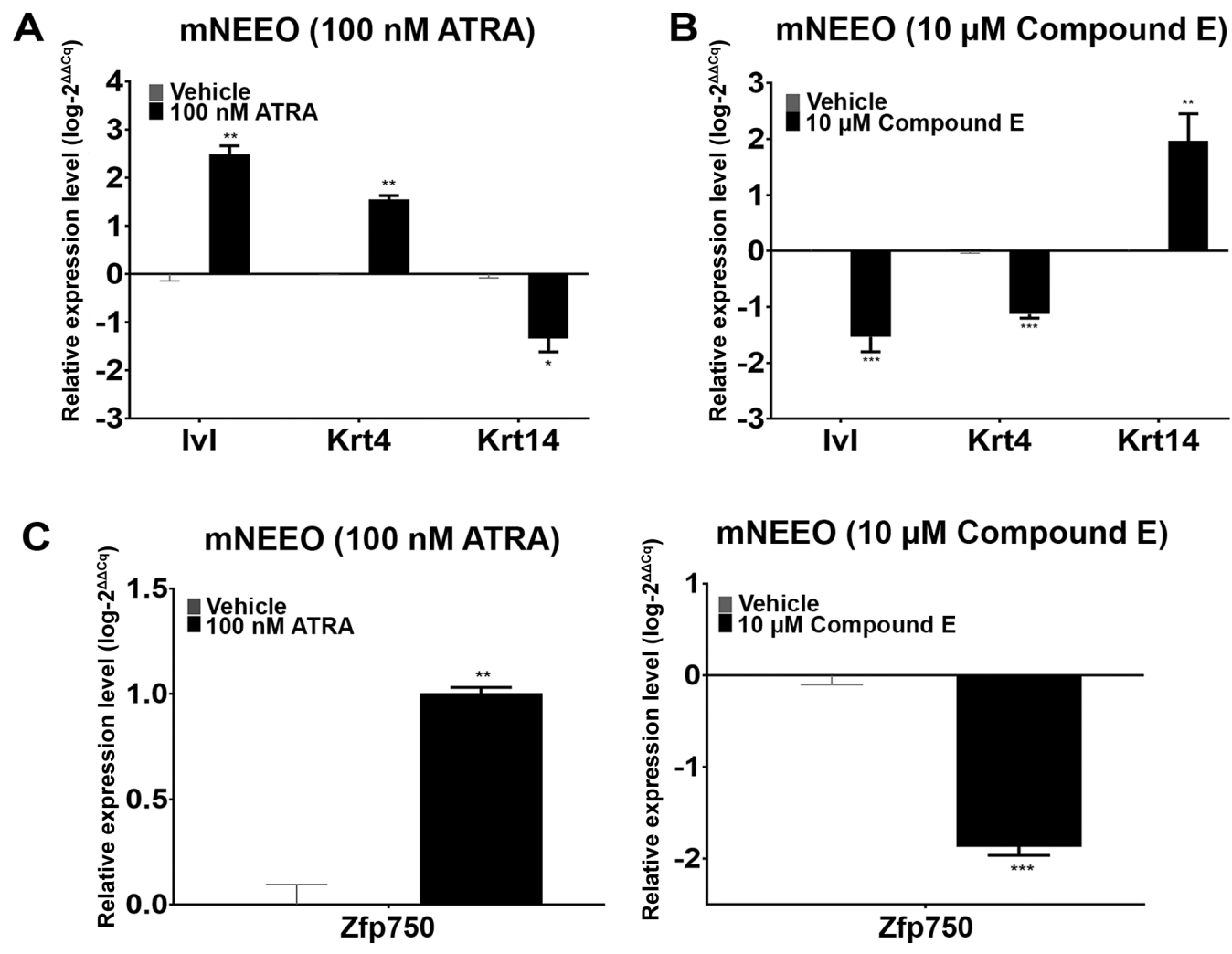

Figure 1. Zfp 750 is involved in the esophageal keratinocyte differentiation program in mNEEO cells. (A) ATRA treatment induced the upregulation of $I v l$ and Krt4 and the downregulation of Krt14 expression levels compared with those in the vehicle-treated cells. (B) Compound E treatment resulted in the downregulation of $\mathrm{Ivl}$ and $\mathrm{Krt} 4$ and upregulation of $\mathrm{Krtl} / \mathrm{expression}$ levels compared with those in the vehicle-treated cells. (C) Zfp750 expression was upregulated upon induction of differentiation through ATRA treatment and downregulated upon inhibition of differentiation through Compound E treatment. Data are presented as the mean $\pm \mathrm{SEM} .{ }^{*} \mathrm{P}<0.05,{ }^{* *} \mathrm{P}<0.01$ and ${ }^{* * *} \mathrm{P}<0.001$ vs. vehicle. mNEEO, mouse normal esophageal organoid; Zfp 750 , zinc finger protein 750 ; ATRA, all-trans retinoic acid; Ivl, involucrin; Krt, keratin.

\section{Results}

Zfp750 is involved in the esophageal keratinocyte differentiation program in mouse epithelial cells. mNEEOs were used to determine the role of $Z f p 750$ in the esophageal keratinocyte differentiation program. Following ATRA treatment, the organoids demonstrated increased expression levels of Ivl and Krt4 and decreased levels of Krt14 compared with those in the vehicle-treated mNEEOs (Fig. 1A), suggesting an induction of the keratinocyte differentiation program and subsequent decrease in the basal cell layers within the organoid. By contrast, following Compound E treatment, the expression levels of $\mathrm{Ivl}$ and $\mathrm{Krt} 4$ were markedly decreased, whereas those of $\mathrm{Krt} 14$ were increased compared with those in the vehicle-treated group (Fig. 1B), suggesting the inhibition of the differentiation program and an increase in the basal cell layers in these organoids. Changes in Zfp 750 expression corresponded with the expression levels of the markers of differentiation, $I v l$ and $K r t 4$, after both treatments (Fig. 1C). These results suggested that $Z f p 750$ expression may be proportionally affected by the stimulation and inhibition of the esophageal keratinocyte differentiation program, and that $Z f p 750$ may be involved in this cellular process in esophageal keratinocytes.

High ZNF750 expression in ESCC tumors is associated with the enrichment of keratinocyte and epidermal cell differentiation gene sets. To determine whether ZNF750 expression may affect the keratinocyte differentiation program in ESCC tumors, RNA-seq data of ESCC tumor samples $(n=81)$ from the TCGA-ESCA dataset were analyzed using GSEA. The samples were stratified into ZNF750-high [median, 20.07; interquartile range (IQR), 19.03-21.11] and ZNF750-low (median, 17.86; IQR, 16.45-19.27) groups (Fig. 2A). The results of GSEA confirmed that epithelial and epidermal differentiation-related cellular processes including 'cornification', 'keratinization', 'keratinocyte differentiation' and 'epidermal cell differentiation' (Table SI) were enriched in the ZNF750-high group compared with the ZNF750-low group. 'Keratinocyte differentiation' and 'epidermal cell differentiation' gene sets were among the most enriched sets (Fig. 2B), whereas SPRR, TGM and $I V L$ were among the most enriched genes (Table SII). These results supported the involvement of ZNF750 in keratinocyte differentiation in ESCC.

ZNF750 overexpression suppresses ESCC cell viability and growth in vitro and in vivo. ZNF750 expression levels were downregulated in the ESCC cell line models compared with those in the immortalized human esophageal epithelial cell line NE1 (Fig. 3A); therefore, to study the suppressive function of ZNF750 in ESCC, ZNF750 was overexpressed in KYSE30 and KYSE180 cells by lentiviral transduction of pLVX-GFP and pLVX-ZNF750. These cell lines were selected as the models for further experiments as they exhibited decreased expression 

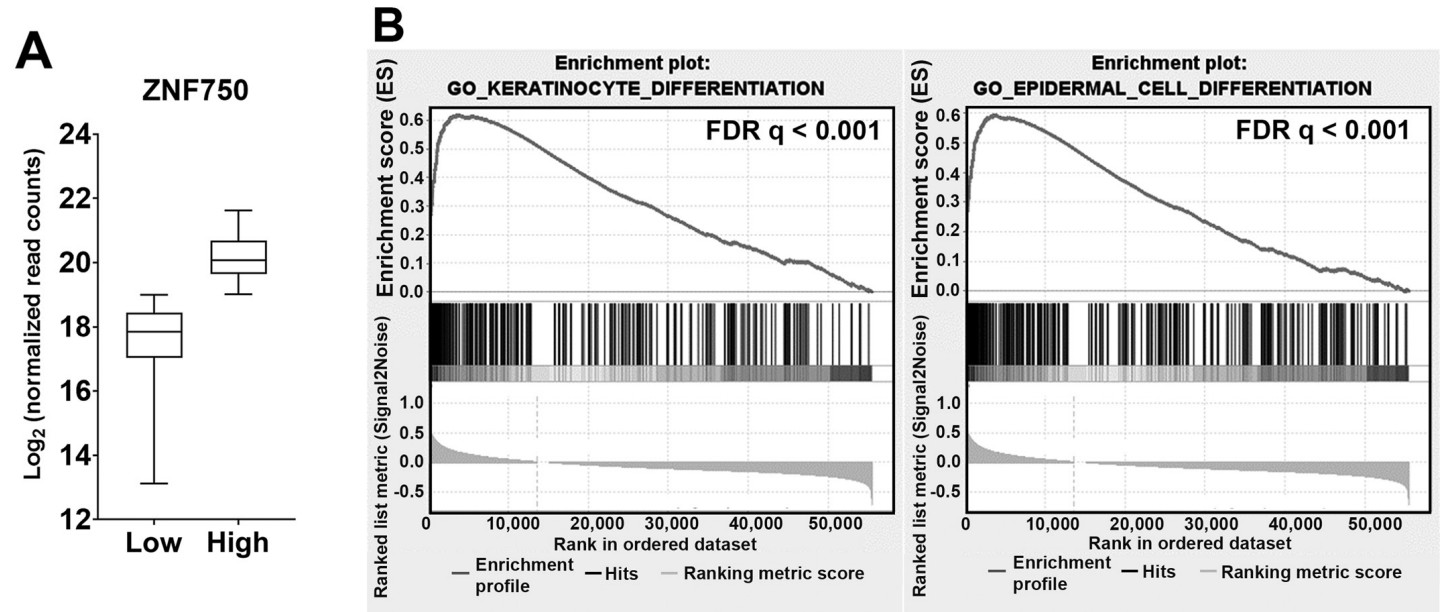

Figure 2. ESCC tumors with high ZNF750 expression levels in The Cancer Genome Atlas exhibit upregulated genes involved in keratinocyte and epidermal differentiation. (A) ZNF750 expression in ESCC tumors with normalized to the median expression as the cut-off. The box and whisker plot represents the normalized median expression and the interquartile range. (B) Enrichment plots of 'keratinocyte differentiation' and 'epidermal cell differentiation' gene sets in the ZNF750-high group compared with the ZNF750-low group. ZNF750, zinc finger protein 750; ESCC, esophageal squamous cell carcinoma.
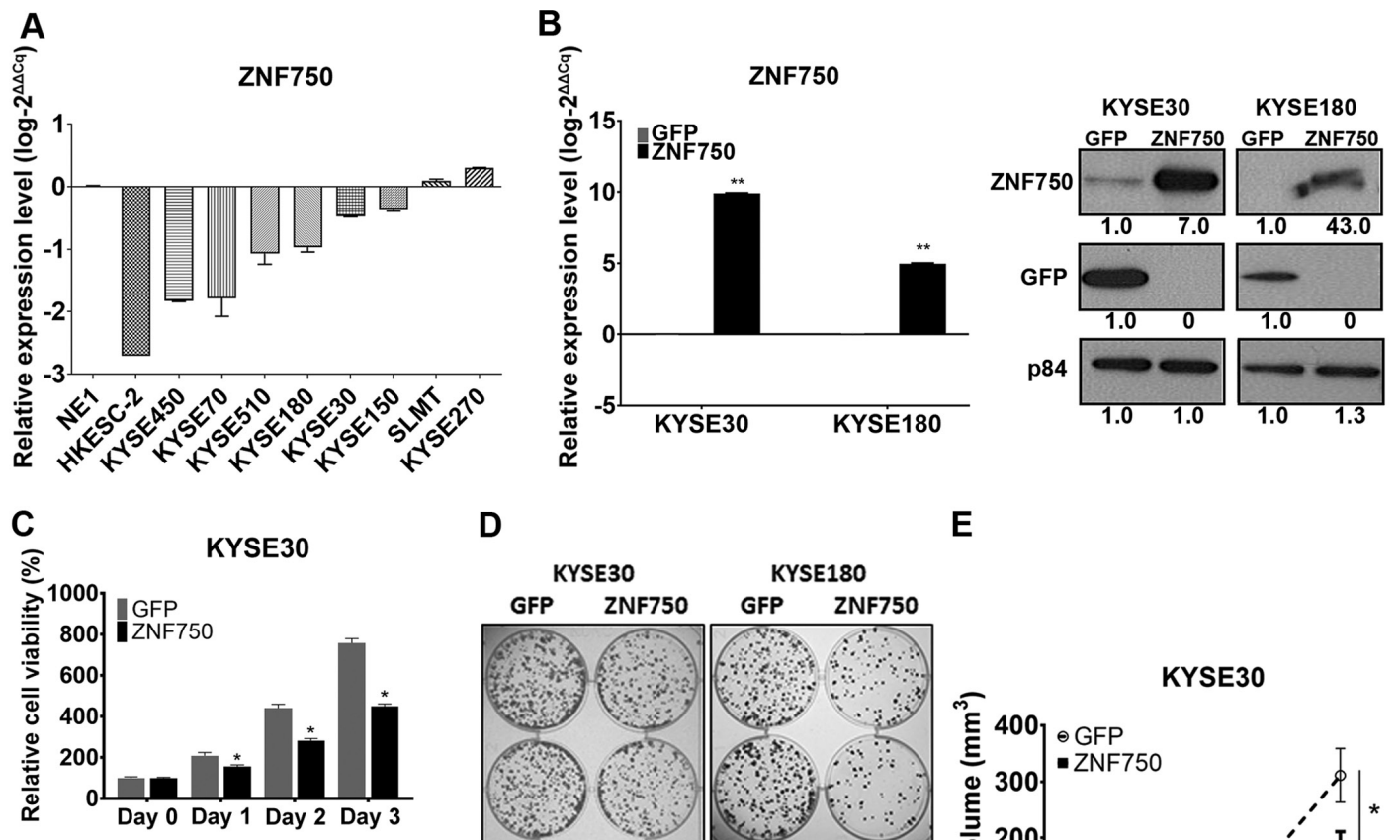

D
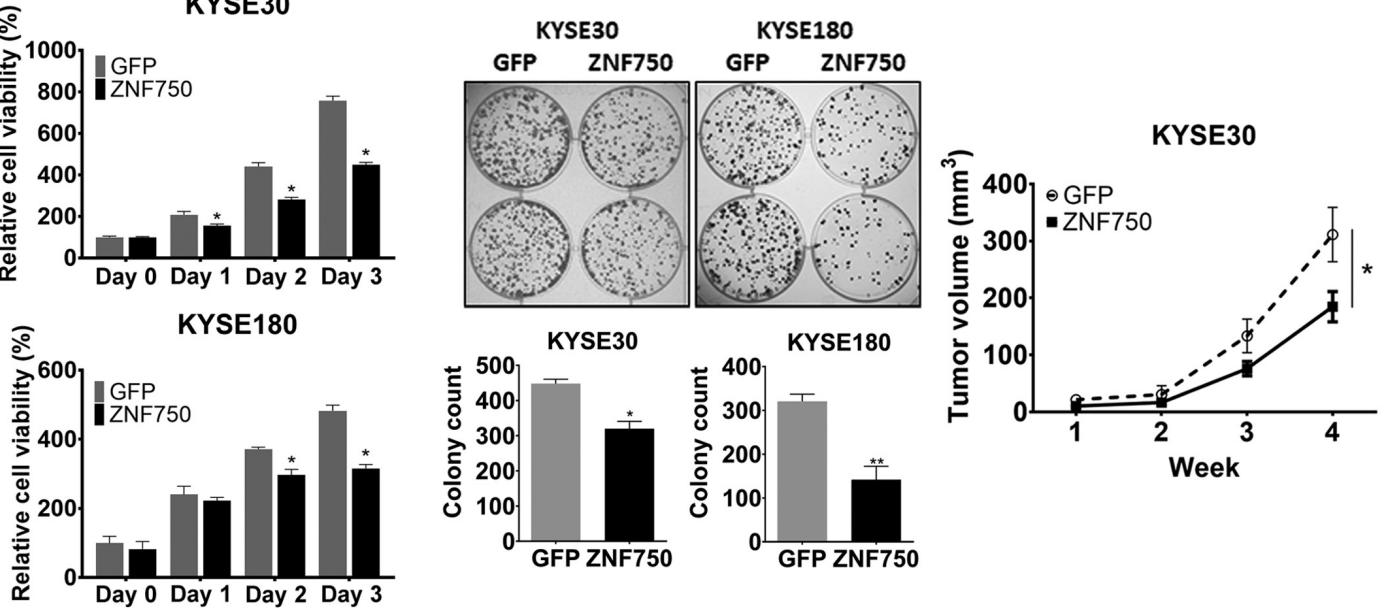

Figure 3. Overexpression of ZNF750 suppresses ESCC cell viability and proliferation in vitro and in vivo. (A) ZNF750 expression levels were downregulated in the majority of the tested ESCC cell lines compared with those in the immortalized normal esophageal cell line NE1. (B) ZNF750 overexpression in KYSE30 and KYSE180 cells was confirmed by reverse transcription-quantitative PCR and western blotting. Numbers under the blots represent quantified band intensities normalized to the GFP control for each presented blot. (C) Overexpression of ZNF750 decreased ESCC cell viability, as determined by the MTT assay. (D) Colony formation assay demonstrated that overexpression of ZNF750 decreased the colony formation ability of ESCC cells compared with that of the control cells. (E) In vivo tumor formation assay in nude mice demonstrated that ZNF750 overexpression inhibited ESCC tumorigenicity. Data are presented as the mean \pm SEM. ${ }^{*} \mathrm{P}<0.05,{ }^{* * *} \mathrm{P}<0.01$ vs. GFP. ZNF750, zinc finger protein 750 ; GFP, control green fluorescent protein lentivirus; ESCC, esophageal squamous cell carcinoma.

of ZNF750 at the mRNA and protein level (Fig. 3A and B). ZNF750 expression levels were upregulated in both cell lines in the pLVX-ZNF750 groups compared with those in the corresponding pLVX-GFP groups, as demonstrated 

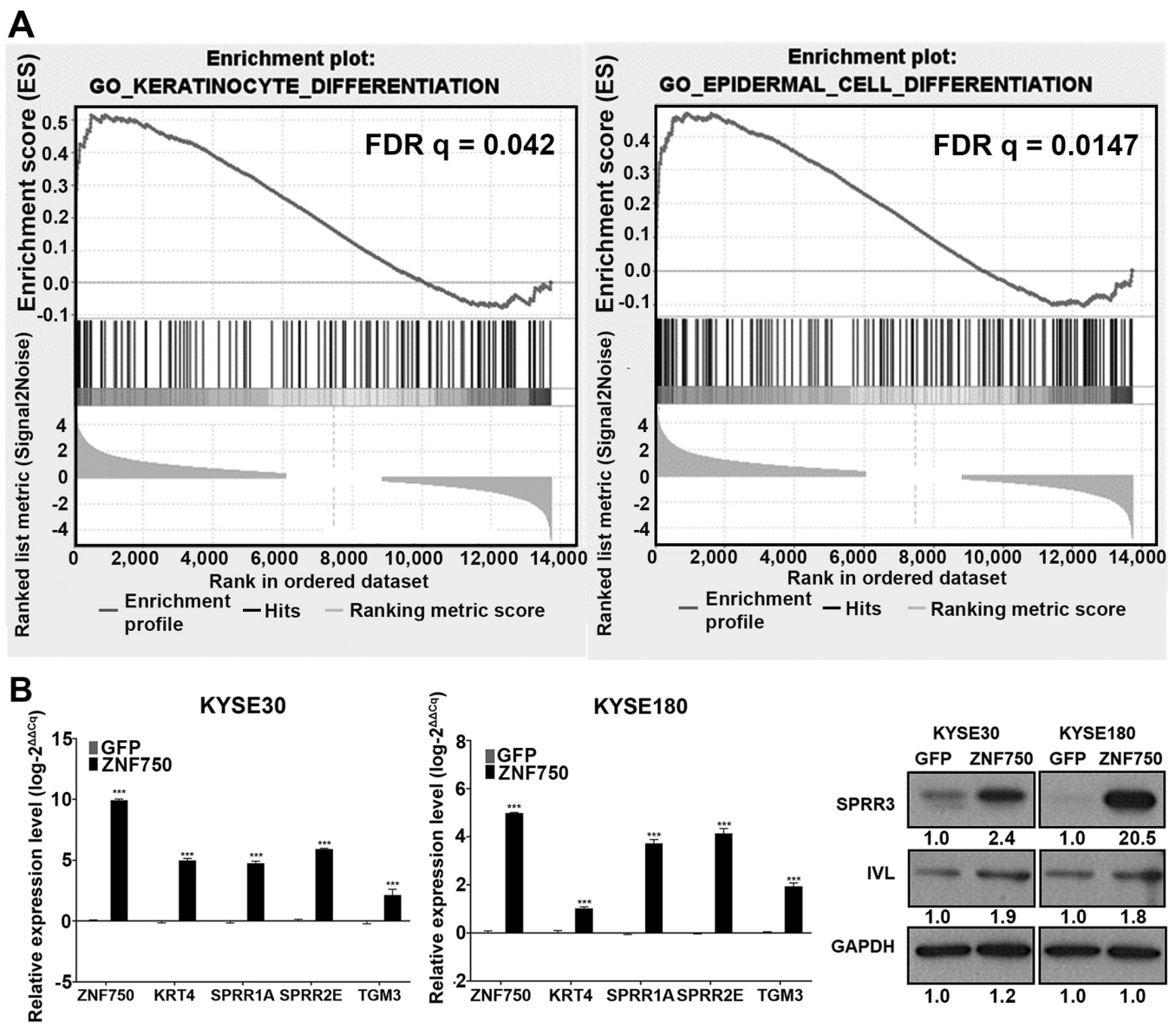

Figure 4. Overexpression of ZNF750 leads to enrichment of differentiation-related gene sets in ESCC cells. (A) Enrichment plots of 'keratinocyte differentiation' and 'epidermal cell differentiation' gene sets analyzed by Gene Set Enrichment Analysis and Toppfun with a false discovery rate $<0.25$. (B) Reverse transcription-quantitative PCR and western blot analyses confirmed the upregulation of differentiation-related gene KRT4, SPRR1A, SPRR2E, SPRR3, TGM3 and $I V L$ expression levels in ZNF750-overexpressing ESCC cells compared with those in the control group. Numbers represent band intensities normalized to the GFP control for the presented blot. Data are presented as the mean \pm SEM. ${ }^{* * *} \mathrm{P}<0.001$ vs. GFP. ZNF750, zinc finger protein 750; GFP, control green fluorescent protein lentivirus; ESCC, esophageal squamous cell carcinoma; SPRR, small proline-rich protein; TGM3, transglutaminase 3; IVL, involucrin.

by RT-qPCR and western blot analyses (Fig. 3B). In vitro MTT cell viability and colony formation assays and in vivo subcutaneous tumorigenicity model were used to assess the tumor-suppressive ability of ZNF750. In vitro, ZNF750 overexpression suppressed ESCC cell viability compared with that in the control group (Fig. 3C). Colony formation ability was also reduced in the ZNF750-overexpressing groups compared with that in the controls (Fig. 3D). In the in vivo subcutaneous tumor model, mice in the ZNF750 overexpression group developed smaller tumors $\left(\mathrm{n}=8\right.$; mean \pm SEM, $\left.184 \pm 26.5 \mathrm{~mm}^{3}\right)$ in mice compared to the GFP control $(n=8$; mean \pm SEM, $\left.311.17 \pm 47.76 \mathrm{~mm}^{3}\right)(\mathrm{P}<0.05$; Fig. 3E). These results demonstrate the ability of ZNF750 to suppress cell viability and growth in vitro and in vivo.

ZNF750 overexpression in ESCC cells induces the expression of esophageal keratinocyte differentiation genes. To determine the pathways affected by ZNF750 overexpression in the in vitro model, ZNF750 and GFP lentivirus-transduced KYSE180 cells were prepared for RNA-seq and subjected to subsequent bioinformatics analyses. The results of the analyses using the MSigDB revealed enrichment of gene sets involved in epithelial differentiation including 'epithelial cell differentiation', 'epidermal cell differentiation' and 'keratinocyte differentiation' (Table SIII). Consistent with the GSEA analysis of ZNF750-high ESCC tumors, these results confirmed the enrichment of keratinocyte differentiation and epidermal cell differentiation gene sets in ZNF750-overexpressing ESCC cells compared with the controls (Fig. 4A). The upregulation of differentiation-related genes KRT4, SPRRIA, SPRR2E, $T G M 3, I V L$ and $S P R R 3$ were validated by RT-qPCR or western blotting (Fig. 4B). These results suggested that ZNF750 overexpression induced the expression of differentiation-related genes in the ESCC cell line model.

ATRA-induced terminal differentiation decreases the viability of ESCC cells. To determine the effects of the induction of the keratinocyte differentiation program on ESCC cell viability, parental KYSE30 and KYSE180 cells were treated with ATRA and subsequently tested for changes in cell viability using the MTT assay. The results demonstrated a significant suppression in the viability of the ATRA-treated parental ESCC cells compared with that in the vehicle-treated group at $48 \mathrm{~h}$ post-treatment (Fig. 5A). RT-qPCR analysis revealed that ATRA treatment upregulated the expression levels of $I V L$ compared with those in the vehicle group (Fig. 5B), indicating the induction of terminal differentiation. These results suggested that induction of the keratinocyte differentiation 
A
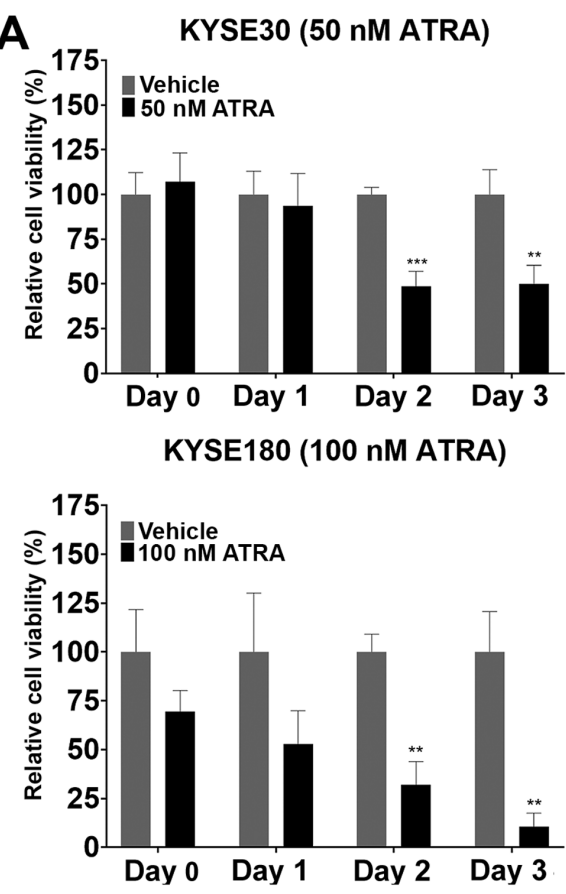

B

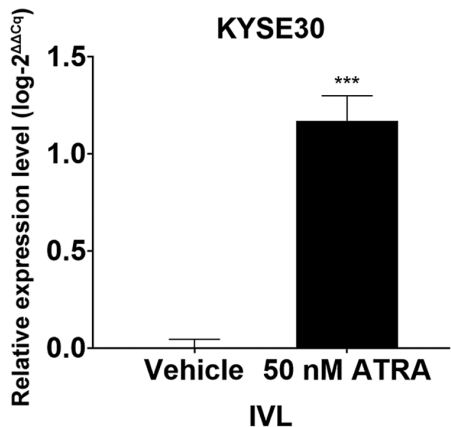

IVL

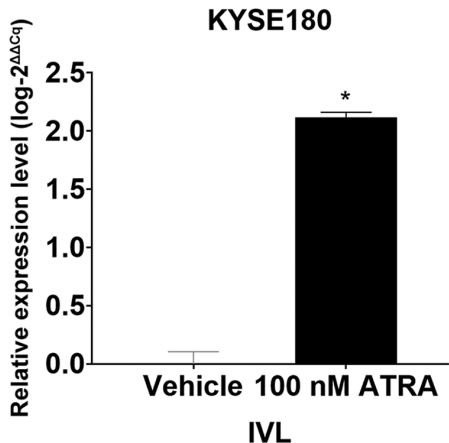

Figure 5. Induction of keratinocyte differentiation through to terminal differentiation contributes to suppression of ESCC cell viability. (A) MTT assay of KYSE30 and KYSE180 cells demonstrated the suppression of ESCC cell viability following ATRA treatment. (B) Reverse transcription-quantitative PCR analysis of ESCC cells treated with ATRA for $48 \mathrm{~h}$ revealed significant upregulation of the mRNA levels of the terminal differentiation marker $I V L$. Data are presented as the mean \pm SEM. ${ }^{*} \mathrm{P}<0.05,{ }^{* *} \mathrm{P}<0.01$ and ${ }^{* * *} \mathrm{P}<0.001$ vs. vehicle. ATRA, all-trans retinoic acid; $I V L$, involucrin.

program through to terminal differentiation may contribute to the suppression of ESCC cell viability following ATRA-induced stimulation of differentiation.

\section{Discussion}

ESCC is a highly malignant SCC of the esophagus (29). Although SCCs arise from various organ systems, they have been reported to share common genetic, genomic and epigenetic modifications relevant to malignancy, and the development of SCCs is associated with the deregulation of squamous cell lineage commitment that may ultimately lead to carcinogenesis (3). Numerous landscape studies have aimed to identify the genetic and genomic changes that contribute to the development of $\operatorname{ESCC}(9,14,30,31)$. These studies identified frequently mutated genes in ESCC including common cancer-related (TP53, RB1, CDKN2A, PIK3CA, NOTCH1 and NFE2L2) and previously unreported (ADAM29, FAM135B, ZNF750 and FAT1) Genes. ZNF750 is a gene that encodes a transcription factor involved in the terminal differentiation of SCCs $(8,14)$ and has been reported to be one of the most frequently mutated genes in ESCC, suggesting that it may function as a differentiation-related tumor suppressor gene. Although in vitro and in vivo studies have provided evidence for ZNF750 functioning as a tumor suppressor and as an inducer of differentiation in $\operatorname{ESCC}(9,14)$, the link between the functions of ZNF750 in inducing differentiation and tumor suppression in ESCC is yet to be fully explored.

The results of the present study demonstrated the involvement of $Z f p 750$ in the esophageal keratinocyte differentiation program through the upregulation and downregulation of Zfp750 expression with chemical stimulation and inhibition, by ATRA and Compound E, respectively, of the differentiation program in mNEEOs. ATRA is a chemical promoter of keratinocyte differentiation in esophageal cells $(28,29)$ and was used in the present study to induce the epithelial differentiation program. Alternatively, $\gamma$-secretase inhibitor Compound $\mathrm{E}$ was used to mimic the inhibition of the keratinocyte differentiation program through the inhibition of the NOTCH signaling pathway $(30,31)$. Through the analysis of public data, the present study also confirmed the ability of ZNF750 to induce differentiation-related gene expression through the enrichment of differentiation-related gene sets in ZNF750-high ESCC tumors. In addition, the results of the present study demonstrated that ZNF750 suppressed ESCC cell viability and proliferation in vitro, and its overexpression resulted in smaller tumors in the mouse model compared with those in the corresponding controls. RNA-seq study of the ESCC cell line model further confirmed the involvement of ZNF750 in keratinocyte differentiation in ESCC cells through the upregulation of differentiation-related genes SPRRIA, SPRR2E, SPRR3, TGM3, KRT4 and IVL. Finally, chemically induced stimulation of keratinocyte differentiation through to terminal differentiation by ATRA functionally suppressed ESCC through the observed decrease in cell viability in ATRA-treated cells compared with the controls. This decrease in cell viability was similar to that of ZNF750-overexpressing ESCC cells. Collectively, these results demonstrated the ability of ZNF750 to induce ESCC cell differentiation through to terminal differentiation, as well as the link between this process and the suppression of ESCC cell viability.

The surface of the esophagus is lined with stratified squamous epithelium, which comprises the basal proliferative (keratinocyte) layer that gives rise to the layers of non-dividing 
terminally differentiated cells that form the stratified layer (3). The regulation of this proliferation-differentiation gradient ensures homeostasis within the epithelium (32). The squamous cell commitment to differentiate involves the regulation of the expression levels of genes relevant to this process, and deregulation of this system has been reported to initiate malignancy (33). NOTCH is a major player in epithelial differentiation and one of the most frequently mutated genes in ESCC $(3,9,30)$, suggesting that it may be a candidate tumor suppressor. Differentiation-related genes including SPRR1A, SPRR2E, SPRR3, TGM3 and IVL have been reported to be downregulated in $\operatorname{ESCC}(11,34)$. SPRR3, also termed esophagin, is one of the genes upregulated by ZNF750 in ESCC cells in the current study, and has been implicated as a tumor suppressor in ESCC (13). Exogenous expression of SPRR3 attenuates ESCC tumorigenicity by promoting apoptosis (13) and enhances sensitization to DNA damage-related apoptosis (35). In addition, TGM3 has been reported as a potential prognostic marker in ESCC (12). Proteomics analysis in tissues from patients with ESCC has revealed that the presence of TGM3 in ESCC tissues is correlated with a longer survival time (12). These results suggest that epithelial differentiation-related genes are downregulated or silenced in ESCC and may function as putative tumor suppressors or prognostic markers, providing evidence of the relevance of this process in ESCC development.

The ability of ZNF750 to induce keratinocyte terminal differentiation indicated by the expression of $I V L$ in the present study resembled the chemically induced differentiation stimulation that caused increased IVL expression in ESCC cell lines. ESCC viability suppression was consistently observed in ZNF750-overexpressing and differentiation-induced cells, suggesting a functional association between the two groups. This provides a novel functional indication of the link between the differentiation-related functions of ZNF750 and its tumor-suppressive ability in ESCC.

In conclusion, the results of the present study demonstrated that ZNF750 may be a differentiation-related silenced tumor suppressor gene in ESCC. Overexpression of ZNF750 in ESCC cells led to the upregulation of differentiation-related genes that may restore epithelial homeostasis by driving differentiation through to terminal differentiation and, thus, restoring the proliferation-differentiation balance. In addition, the ability of ZNF750 to induce the expression of genes such as SPRR3, which exhibit tumor-suppressive functions in ESCC, may contribute to its ability to suppress ESCC. Further studies are required to fully elucidate the link between the differentiation-related functions of ZNF750 and its growth-suppressive ability in ESCC.

\section{Acknowledgements}

The authors would like to thank Dr Wei Dai (Department of Clinical Oncology, The University of Hong Kong) for insights and guidance with bioinformatics analysis. The authors would also like to thank Professor George Tsao (School of Biomedical Sciences) and Professor Gopesh Srivastava (Department of Pathology) of The University of Hong Kong for providing the cell lines used in the study.

\section{Funding}

This work was funded by the Research Grants Council Collaborative Research Fund (grant no. 106150246), the Theme-based Research Scheme (grant no. T12-701/17-R) and the Asian Cancer Research Fund.

\section{Availability of data and materials}

The RNA sequencing data generated and/or analyzed during the current study are available in the National Center for Biotechnology Information Sequence Read Archive (https://www.ncbi.nlm.nih.gov/sra) under the accession no. PRJNA707329.

\section{Authors' contributions}

SSAC contributed to the design and execution of the study and data interpretation, and wrote the manuscript. JMYK and VZY contributed to the design of the study, data interpretation and the preparation of the manuscript. SSAC and JMYK confirm the authenticity of the raw data. LN contributed to the processing of the raw RNA-sequencing data and its subsequent analysis. MLL contributed to the study design, preparation of the manuscript and overall supervision of the study. All authors have read and approved the final manuscript.

\section{Ethics approval and consent to participate}

Ethics approval for animal studies was obtained from the Animal Ethics Committee of The University of Hong Kong (approval no. CULATR 3377-14). The tumor burden did not exceed the recommended dimensions set by The University of Hong Kong, and the animals were anesthetized and sacrificed using acceptable methods/techniques.

\section{Patient consent for publication}

Not applicable.

\section{Competing interests}

The authors declare that they have no competing interests.

\section{References}

1. Ferlay J, Colombet M, Soerjomataram I, Mathers C, Parkin DM, Piñeros M, Znaor A and Bray F: Estimating the global cancer incidence and mortality in 2018: GLOBOCAN sources and methods. Int J Cancer 144: 1941-1953, 2019.

2. Abnet CC, Arnold M and Wei WQ: Epidemiology of esophageal squamous cell carcinoma. Gastroenterology 154: 360-373, 2018

3. Dotto GP and Rustgi AK: Squamous cell cancers: A unified perspective on biology and genetics. Cancer Cell 29: 622-637, 2016.

4. Cassandri M, Smirnov A, Novelli F, Pitolli C, Agostini M, Malewicz M, Melino G and Raschellà G: Zinc-finger proteins in health and disease. Cell Death Discov 3: 17071, 2017.

5. Jen J and Wang YC: Zinc finger proteins in cancer progression. J Biomed Sci 23: 53, 2016.

6. Sen GL, Boxer LD, Webster DE, Bussat RT, Qu K, Zarnegar BJ, Johnston D, Siprashvili Z and Khavari PA: ZNF750 is a p63 target gene that induces KLF4 to drive terminal epidermal differentiation. Dev Cell 22: 669-677, 2012. 
7. Boxer LD, Barajas B, Tao S, Zhang J and Khavari PA: ZNF750 interacts with KLF4 and RCOR1, KDM1A, and CTBP1/2 chromatin regulators to repress epidermal progenitor genes and induce differentiation genes. Genes Dev 28: 2013-2026, 2014.

8. Hazawa M, Lin DC, Handral H, Xu L, Chen Y, Jiang YY, Mayakonda A, Ding LW, Meng X, Sharma A, et al: ZNF750 is a lineage-specific tumour suppressor in squamous cell carcinoma. Oncogene 36: 2243-2254, 2017.

9. Zhang L, Zhou Y, Cheng C, Cui H, Cheng L, Kong P, Wang J, Li Y, Chen W, Song B, et al: Genomic analyses reveal mutational signatures and frequently altered genes in esophageal squamous cell carcinoma. Am J Hum Genet 96: 597-611, 2015.

10. Dai W, Ko JMY, Choi SSA, Yu Z, Ning L, Zheng H, Gopalan V, Chan KT, Lee NP, Chan KW, et al: Whole-exome sequencing reveals critical genes underlying metastasis in oesophageal squamous cell carcinoma. J Pathol 242: 500-510, 2017.

11. Luo A, Kong J, Hu G, Liew CC, Xiong M, Wang X, Ji J, Wang T, Zhi $\mathrm{H}, \mathrm{Wu} \mathrm{M}$ and Liu Z: Discovery of $\mathrm{Ca}^{2+}$-relevant and differentiation-associated genes downregulated in esophageal squamous cell carcinoma using cDNA microarray. Oncogene 23: 1291-1299, 2004

12. Uemura N, Nakanishi Y, Kato H, Saito S, Nagino M, Hirohashi S and Kondo T: Transglutaminase 3 as a prognostic biomarker in esophageal cancer revealed by proteomics. Int J Cancer 124: 2106-2115, 2009.

13. Zhang Y, Feng YB, Shen XM, Chen BS, Du XL, Luo ML, Cai Y, Han YL, Xu X, Zhan QM and Wang MR: Exogenous expression of Esophagin/SPRR3 attenuates the tumorigenicity of esophageal squamous cell carcinoma cells via promoting apoptosis. Int J Cancer 122: 260-266, 2008.

14. Lin DC, Hao JJ, Nagata Y, Xu L, Shang L, Meng X, Sato Y, Okuno Y, Varela AM, Ding LW, et al: Genomic and molecular characterization of esophageal squamous cell carcinoma. Nat Genet 46: 467-473, 2014

15. DeWard AD, Cramer J and Lagasse E: Cellular heterogeneity in the mouse esophagus implicates the presence of a nonquiescent epithelial stem cell population. Cell Rep 9: 701-711, 2014.

16. Sanger F, Nicklen S and Coulson AR: DNA sequencing with chain-terminating inhibitors. Proc Natl Acad Sci USA 74 5463-5467, 1977

17. Shuen WH, Kan R, Yu Z, Lung HL and Lung ML: Nove lentiviral-inducible transgene expression systems and versatile single-plasmid reporters for in vitro and in vivo cancer biology studies. Cancer Gene Ther 22: 207-214, 2015.

18. Livak KJ and Schmittgen TD: Analysis of relative gene expression data using real-time quantitative PCR and the 2(-Delta Delta C(T)) method. Methods 25: 402-408, 2001.

19. Schneider CA, Rasband WS and Eliceiri KW: NIH Image to ImageJ: 25 years of image analysis. Nat Methods 9: 671-675, 2012.

20. Kim D, Pertea G, Trapnell C, Pimentel H, Kelley R and Salzberg SL: TopHat2: Accurate alignment of transcriptomes in the presence of insertions, deletions and gene fusions. Genome Biol 14: R36, 2013.

21. Trapnell C, Roberts A, Goff L, Pertea G, Kim D, Kelley DR, Pimentel H, Salzberg SL, Rinn JL and Pachter L: Differential gene and transcript expression analysis of RNA-seq experiments with TopHat and Cufflinks. Nat Protoc 7: 562-578, 2012.
22. Subramanian A, Tamayo P, Mootha VK, Mukherjee S, Ebert BL, Gillette MA, Paulovich A, Pomeroy SL, Golub TR, Lander ES and Mesirov JP: Gene set enrichment analysis: A knowledge-based approach for interpreting genome-wide expression profiles. Proc Natl Acad Sci USA 102: 15545-15550, 2005.

23. Chen J, Bardes EE, Aronow BJ and Jegga AG: ToppGene Suite for gene list enrichment analysis and candidate gene prioritization. Nucleic Acids Res 37: 305-311, 2009.

24. Grossman RL, Heath AP, Ferretti V, Varmus HE, Lowy DR, Kibbe WA and Staudt LM: Toward a shared vision for cancer genomic data. N Engl J Med 375: 1109-1112, 2016.

25. Collins FS and Barker AD: Mapping the cancer genome Pinpointing the genes involved in cancer will help chart a new course across the complex landscape of human malignancies. Sci Am 296: 50-57, 2007.

26. R Core Team (2014). R: A language and environment for statistical computing. R Foundation for Statistical Computing, Vienna, Austria. URL http://www.R-project.org/.

27. Colaprico A, Silva TC, Olsen C, Garofano L, Cava C, Garolini D, Sabedot TS, Malta TM, Pagnotta SM, Castiglioni I, et al: TCGAbiolinks: An R/Bioconductor package for integrative analysis of TCGA data. Nucleic Acids Res 44: e71, 2016.

28. Koterazawa Y, Koyanagi-Aoi M, Uehara K, Kakeji Y and Aoi T: Retinoic acid receptor $\gamma$ activation promotes differentiation of human induced pluripotent stem cells into esophageal epithelium. J Gastroenterol 55: 763-774, 2020.

29. Ohashi S, Miyamoto S, Kikuchi O, Goto T, Amanuma Y and Muto M: Recent advances from basic and clinical studies of esophageal squamous cell carcinoma. Gastroenterology 149: 1700-1715, 2015.

30. Song Y, Li L, Ou Y, Gao Z, Li E, Li X, Zhang W, Wang J, Xu L, Zhou Y, et al: Identification of genomic alterations in oesophageal squamous cell cancer. Nature 509: 91-95, 2014

31. Gao YB, Chen ZL, Li JG, Hu XD, Shi XJ, Sun ZM, Zhang F, Zhao ZR, Li ZT, Liu ZY, et al: Genetic landscape of esophageal squamous cell carcinoma. Nat Genet 46: 1097-1102, 2014.

32. Whelan KA, Muir AB and Nakagawa $\mathrm{H}$ : Esophageal 3D culture systems as modeling tools in esophageal epithelial pathobiology and personalized medicine. Cell Mol Gastroenterol Hepatol 5: 461-478, 2018

33. He H, Li S, Hong Y, Zou H, Chen H, Ding F, Wan Y and Liu Z: Krüppel-like factor 4 promotes esophageal squamous cell carcinoma differentiation by Up-regulating keratin 13 expression. J Biol Chem 290: 13567-1377, 2015.

34. Zhong X, Huang G, Ma Q, Liao H, Liu C, Pu W, Xu L, Cai Y and Guo X: Identification of crucial miRNAs and genes in esophageal squamous cell carcinoma by miRNA-mRNA integrated analysis. Medicine (Baltimore) 98: e16269, 2019.

35. Luo A, Chen H, Ding F, Zhang Y, Wang M, Xiao Z and Liu Z: Small proline-rich repeat protein 3 enhances the sensitivity of esophageal cancer cells in response to DNA damage-induced apoptosis. Mol Oncol 7: 955-967, 2013. 